For reprint orders, please contact: reprints@futuremedicine.com

\title{
Fentanyl iontophoretic transdermal system versus morphine intravenous patient-controlled analgesia for pain management following gynecological surgery: a meta-analysis of randomized, controlled trials
}

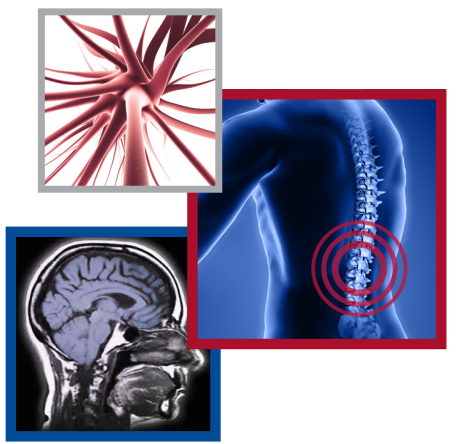

\author{
Craig S Saffer ${ }^{1}$, Harold S Minkowitz ${ }^{2}$, Li Ding ${ }^{3}$, Hassan Danesi ${ }^{* 3}$ \& James B Jones ${ }^{3}$
}

Practice points

- Patients and investigators had higher ratings on the patient global assessment of pain control and on the investigator global assessment of pain control, respectively, of fentanyl iontophoretic transdermal system compared with morphine intravenous patient-controlled analgesia as a method of postoperative pain control following gynecological surgery.

- The most frequently occurring treatment-emergent adverse events in both treatment groups were nausea, headache and pyrexia.

- The Hospital Consumer Assessment of Healthcare Providers and Systems (HCAHPS) incorporates queries regarding the level of pain and efforts made to control pain; this expanded focus on pain management and its incorporation into overall patient satisfaction may reward the use of new technologies like the fentanyl iontophoretic transdermal system, which have high ratings in patient-reported outcomes extending beyond level of pain.

Aim: To compare the efficacy and safety of patient-controlled fentanyl iontophoretic transdermal system (ITS) with morphine intravenous (iv.) patient-controlled analgesia (PCA) for pain management following gynecological surgery. Methods: Two-openlabel, multicenter, randomized, active-controlled, parallel-group studies $(n=1142)$ were conducted that compared fentanyl ITS with morphine iv. PCA for postoperative pain. The subgroup of gynecological surgery patients from each trial was utilized for this metaanalysis ( $n=604)$. Of these patients, 295 received fentanyl ITS ( $40 \mu \mathrm{g} / \mathrm{dose})$ and 309 received morphine iv. PCA (1 mg/dose) for up to $72 \mathrm{~h}$. Efficacy measures included the patient global assessment (PGA) and the investigator global assessment (IGA) of the method of pain control. Results: Gynecological surgery patients $(n=604)$ included in this meta-analysis had a mean age of 45 years, were predominantly Caucasian (65\%) and had a mean body mass index of $29 \mathrm{mg} / \mathrm{kg}^{2}$. There were statistically significantly more patients treated with fentanyl ITS and more investigators who rated their pain control method as 'excellent' on the PGA at $24 \mathrm{~h}$ (49.3 vs $37.4 \%$, respectively; $p=0.0029)$ and IGA at the last assessment (59.5 vs $38.0 \%$, respectively; $p<0.0001$ ), respectively, compared with morphine iv. PCA at the last assessment. Conclusion: Following gynecological surgery, patients and investigators were more satisfied (had a higher percent of an 'excellent' rating on the PGA and IGA, respectively) with fentanyl ITS than morphine iv. PCA as a method of pain control. 


\section{KEYWORDS}

- fentanyl • gynecologic surgery $\bullet$ intravenous

- iontophoretic transdermal system $\bullet$ morphine

- patient-controlled analgesia

- postoperative pain
Approximately $75 \%$ of surgical patients experience moderate-to-severe postoperative pain [1,2]. This number has remained steady over the past decade despite advances in technology and availability of analgesics [1,2]. Poorly managed postoperative pain may inhibit mobility which can lead to postoperative complications such as deep vein thrombosis, pulmonary embolus and pneumonia [3]. Patients with postoperative pain also have a tendency to have extended hospital stays as well as more frequent readmissions, which leads to increased healthcare costs [3]. Adequate pain control is the goal for all postoperative patients.

Gynecological surgeries, such as vaginal and abdominal hysterectomies, are commonly performed throughout the world. There are approximately 390,000 hysterectomy procedures performed in the USA annually [4]. Intravenous (iv.) patient-controlled analgesia (PCA) with opioids is commonly utilized to manage moderate-tosevere postoperative pain following gynecological surgery. PGA offers advantages over conventional analgesia which includes improved pain relief, lower opioid consumption, less sedation and greater patient satisfaction $[5,6]$.

Fentanyl is an opioid analgesic that was formulated by Janssen Pharmaceutica in the 1950s and is approximately 50 - to 100 -times more potent than morphine $[7,8]$. Despite being a more potent analgesic, fentanyl tends to induce less histamine-mediated itching, compared with morphine [9]. Fentanyl is highly lipophilic and may be administered through various routes including intravenous, transdermal, transmucosal and inhalation [10]. Depending upon the route of administration, fentanyl can have a very rapid onset of action [11]. Fentanyl is primarily metabolized in the liver by CYP450 3A4 and its metabolites have no relevant activity [12].

The fentanyl iontophoretic transdermal system (ITS; The Medicines Company, NJ, USA) is a self-adhesive, needle-free patient-controlled analgesia that provides systemic delivery of fentanyl to patients for the management of moderate -to-severe pain in the hospital setting. This system works via the process of iontophoresis utilizing a virtually imperceptible low-intensity electric current to repel positively charged fentanyl molecules through the skin's stratum corneum into the subcutaneous space for absorption into the circulatory system. Fentanyl ITS is administered as an on-demand dose of $40 \mu \mathrm{g}$ at steady state delivered over a 10 -min interval. Up to six doses can be self-administered per hour and a maximum of 80 doses (3200 $\mu \mathrm{g})$ per unit per $24 \mathrm{~h}$. Fentanyl ITS has been extensively studied in placebo-controlled and active-comparator trials in hospitalized adult postoperative patients with moderate-to-severe pain [13-18]. In the placebo-controlled trials, fentanyl ITS was superior to placebo in terms of efficacy $[13,16]$. In the active-comparator trials, fentanyl ITS demonstrated similar efficacy and tolerability to morphine iv. PCA $[14,15,17,18]$. The aim of the current meta-analysis was to compare the efficacy and safety of fentanyl ITS with morphine iv. PCA in female patients who had undergone gynecological surgery in two Phase III studies [14,17].

\section{Methods}

Two prospective, randomized, multicenter, openlabel, active-controlled studies were conducted that compared the efficacy and safety of fentanyl ITS with morphine iv. PCA for the management of postoperative pain. Study protocols were approved by the institutional review boards or research ethics boards of each participating center or by an independent centralized ethics research board. Written informed consent was obtained from each patient prior to enrollment in the study. The full study descriptions and results from both studies have been previously published $[14,17]$. These two studies included patients who had undergone gynecological, orthopedic, thoracic or other major abdominal surgeries. The primary efficacy outcome of both studies was the proportion of patients who had success in pain management as assessed using the patient global assessment (PGA) of the method of pain control at the end of the first $24 \mathrm{~h}$ following surgery. The PGA is a 4-point scale where the patient assesses the method of pain control as excellent, good, fair or poor. The PGA is analyzed in terms of success ('good' or 'excellent') or failure ('fair' or 'poor'). The PGA may also be analyzed in terms of 'excellent' versus 'good', 'fair' or 'poor.' In both studies, fentanyl ITS provided patient-controlled analgesia that was therapeutically equivalent to morphine iv. PCA, as assessed via the PGA. For the purposes of this meta-analysis, patients were included if they had undergone gynecologic surgery (Figure 1).

\section{- Patient-controlled systems}

After surgery, if necessary, patients were titrated to an acceptable level of comfort using morphine, hydromorphone, fentanyl, sufentanil or alfentanil. Fentanyl ITS or morphine iv. PCA treatments 


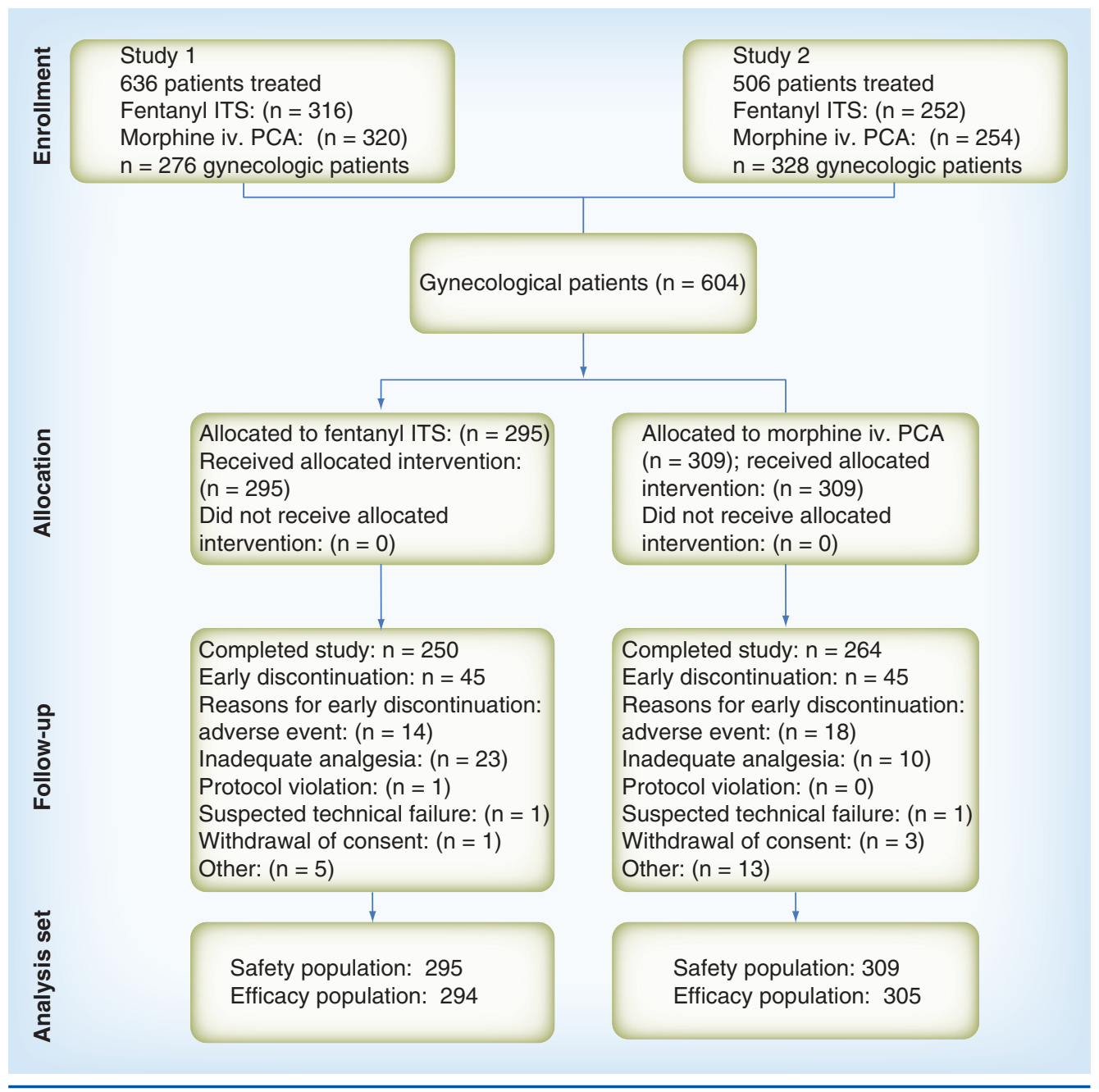

Figure 1. Flowchart of patients.

ITS: Iontophoretic transdermal system; iv.: Intravenous; PCA: Patient-controlled analgesia.

Study 1: data taken from [14].

Study 2: data taken from [17].

were then initiated. Supplemental opioids were permitted only during the first $3 \mathrm{~h}$ of the trial. Patients who received supplemental analgesia greater than $3 \mathrm{~h}$ after enrollment were withdrawn from the study due to inadequate analgesia.

Fentanyl ITS delivered a fixed dose of fentanyl $(40 \mu \mathrm{g})$ over a period of $10 \mathrm{~min}$ upon patient activation. The system was programmed to deliver up to six doses per hour for up to $24 \mathrm{~h}$ or to a maximum of 80 doses, whichever occurred first. Study drug was continued for up to $72 \mathrm{~h}$ if required. The morphine iv. PCA pumps were set to deliver 1-mg boluses on demand with a 5-min lockout interval and a maximum of $10 \mathrm{mg} / \mathrm{h}$. Study drug was continued for up to $72 \mathrm{~h}$ if required. The morphine iv. PCA regimen was considered standard among the many institutions and provided equianalgesic dosing compared with the fentanyl ITS.

\section{- Surgical categories}

For the purposes of this meta-analysis, all surgeries were categorized into three groups: patients with abdominal hysterectomy $(\mathrm{n}=365)$, patients with vaginal hysterectomy $(\mathrm{n}=119)$ and other $(\mathrm{n}=120)$. The other group included all other surgeries that did not involve a hysterectomy: Other includes the following surgeries: oophorectomy, salpingo-oophorectomy, ovarian cystectomy, myomectomy, perineal surgery, tubal reanastomosis, debulking/adhesiolysis and repair of pelvic organ prolapse including anterior repair, posterior repair and sacrocolpopexy. 


\section{- Efficacy assessments}

The primary efficacy end point for this metaanalysis was the proportion of patients who reported 'excellent' pain management on a 4-point categorical PGA at $24 \mathrm{~h}$, the proportion of investigators who reported 'excellent' pain management at study completion and the mean pain intensity score using visual analog scale (VAS) or numerical rating scale (NRS).

The PGA is a 4-point scale where the patient assesses the method of pain control as excellent, good, fair or poor. The rating was completed by the patient at 24,48 and $72 \mathrm{~h}$. The question posed to each patient at each assessment point was "Overall, would you rate this method of pain control during the last $24 \mathrm{~h}$ as being excellent, good, fair or poor?" Investigator global assessment (IGA) was performed at the last assessment before study completion and used the same 4-point scale as the PGA to assess the method of pain control from the investigator's perspective (excellent, good, fair or poor).

In Study 1 [14], the pain intensity was measured on a $100-\mathrm{mm}$ ungraded VAS that ranged from 'no pain' $(0 \mathrm{~mm})$ to 'worst possible pain' $(100 \mathrm{~mm})$. The patient made a vertical mark on the ungraded $100-\mathrm{mm}$ horizontal line to indicate the amount of pain she was experiencing. In Study 2 [17], the pain intensity was measured using a NRS. A member of the investigator's staff read aloud the following question to the patient: "On a scale of $0-10$, where 0 means no pain and 10 means the worst possible pain, rate the pain that you have now." All NRS scores were multiplied by a factor of 10 to standardize the measure for this analysis. Pain intensity was assessed at 0 , $0.5,1,2,3,4,6$ and $8 \mathrm{~h}$ and every $4 \mathrm{~h}$ thereafter.

\section{- Safety assessments}

Safety was assessed via spontaneously reported treatment-emergent adverse events (TEAEs). In addition, all potential opioid-related TEAEs were reviewed. TEAEs classified as opioidrelated were hypoventilation, hypoxia, hypotension, tachycardia, nausea, vomiting, constipation, ileus, urinary retention, somnolence, confusion and pruritus. Events associated with the delivery devices including suspected device failures were recorded.

\section{- Statistical analyses}

Analyses presented in this paper pertain to the subgroup of patients who had gynecological surgeries from two previously reported studies that compared fentanyl ITS to morphine iv. PCA. The evaluable for efficacy population (defined as all randomized patients who received at least $3 \mathrm{~h}$ of treatment with fentanyl ITS or morphine iv. PCA) was used for efficacy outcomes and the safety population (all randomized patients) was used for reporting adverse events.

The meta-analysis was conducted using random effects models which is generally considered to be more conservative. Analyses using random effects models are assumed to be generalizable to the entire patient population. $I^{2}$ values describe the percentage of total variation across studies that is due to heterogeneity rather than chance. $F^{2}$ is calculated from basic results obtained from a typical meta-analysis as $I^{2}=100 \% \times(\mathrm{Q}-\mathrm{df}) / \mathrm{Q}$, where $\mathrm{Q}$ is Cochran's heterogeneity statistic and $\mathrm{df}$ is the degrees of freedom. Negative values of $F^{2}$ are put equal to zero, so that $I^{2}$ lies between 0 and $100 \%$. A value of $0 \%$ indicates no observed heterogeneity, and larger values show increasing heterogeneity.

For the PGA and IGA, odds ratios (ORs) indicating the probability of the outcome to occur for patient receiving fentanyl ITS versus morphine iv. PCA were calculated. 95\% CIs were provided for all parameters. The pain intensity scores were summarized using means. The TEAEs were summarized by frequencies and percentages. Statistical comparisons between treatment groups for pain intensity and TEAEs utilized Fisher's exact test. The statistical software package SAS version 9.2 was used for the analyses.

\section{Results}

\section{- Patients}

From these two studies, a total of 604 patients (fentanyl ITS, $\mathrm{n}=295$; morphine iv. PCA, $\mathrm{n}=309$ ) underwent gynecological surgery (Figure 1). Similar numbers of patients in each treatment group completed the study (fentanyl ITS, $\mathrm{n}=250$ [84.7\%]; morphine iv. PCA, $\mathrm{n}=264[85.4 \%]$ ) (Figure 1). Baseline patient demographics and characteristics were similar between patients in the fentanyl ITS group and the morphine iv. PCA group (Table 1).

\section{- Efficacy outcomes}

Patient assessment of pain management during the first $24 \mathrm{~h}$

There was a statistically significant benefit seen in the fentanyl ITS group compared with the morphine iv. PCA group for the 'excellent' category of the PGA scale (OR: 1.642; 95\% CI: $1.185-$ $2.276 ; \mathrm{p}=0.0029)$ (Table 2). There was a numerical 
Table 1. Baseline characteristics and demographic data, safety population.

\begin{tabular}{|c|c|c|c|c|c|c|c|c|c|}
\hline Surgical category & Treatment & $\mathbf{n}$ & $\begin{array}{l}\text { Age (years); } \\
\text { mean (SD) }\end{array}$ & \multicolumn{2}{|c|}{ Age distribution } & \multicolumn{3}{|c|}{ Race } & $\begin{array}{l}\text { BMI }\left(\mathrm{kg} / \mathrm{m}^{2}\right) ; \\
\text { mean }(\mathrm{SD})\end{array}$ \\
\hline $\begin{array}{l}\text { All gynecological } \\
\text { surgery patients }\end{array}$ & Morphine iv. PCA & 309 & $45.3(10.7)$ & $291(94.2)$ & $18(5.8)$ & $199(64.4)$ & $87(28.2)$ & $23(7.4)$ & $29.9(7.3)$ \\
\hline \multirow{2}{*}{$\begin{array}{l}\text { Abdominal } \\
\text { hysterectomy }\end{array}$} & Fentanyl ITS & 189 & $45.6(10.4)$ & $174(92.1)$ & $15(7.9)$ & $125(66.1)$ & $48(25.4)$ & $16(8.5)$ & $29.4(6.8)$ \\
\hline & Morphine iv. PCA & 176 & $47.1(9.9)$ & $165(93.8)$ & $11(6.3)$ & $121(68.8)$ & $40(22.7)$ & $15(8.5)$ & $30.6(7.8)$ \\
\hline $\begin{array}{l}\text { Vaginal } \\
\text { hysterectomy }\end{array}$ & Morphine iv. PCA & 58 & $44.0(8.8)$ & $57(98.3)$ & $1(1.7)$ & $47(81.0)$ & $9(15.5)$ & $2(3.4)$ & $27.7(6.0)$ \\
\hline \multirow[t]{2}{*}{ Other } & Fentanyl ITS & 45 & $42.3(12.9)$ & $41(91.1)$ & $4(8.9)$ & $21(46.7)$ & $16(35.6)$ & $8(17.7)$ & $30.0(7.9)$ \\
\hline & Morphine iv. PCA & 75 & $41.9(12.8)$ & $69(92.0)$ & $6(8.0)$ & $31(41.3)$ & $38(50.7)$ & $6(8)$ & $29.7(6.7)$ \\
\hline
\end{tabular}

advantage for the primary end point in favor of fentanyl ITS group compared with the morphine iv. PCA group in each surgical category (Table 2).

\section{IGA of method of pain control at last}

assessment

There was a statistically significant advantage for the fentanyl ITS group compared with the morphine iv. PCA group in ratings of 'excellent' overall (OR: 2.413; 95\% CI: 1.736-3.353; $\mathrm{p}<0.0001)$ and in all of the surgical subcategories except vaginal hysterectomy (Table 2).

Pain intensity during the first $24 \mathrm{~h}$

The mean pain intensity scores (measured on either the VAS or the NRS) improved compared with baseline in both treatment groups at each measured time point (Figure 2). Pain intensity scores tended to start at around 40 at study initiation and dropped by approximately 10-12 points by the end of $24 \mathrm{~h}$.

\section{- Safety outcomes}

The most frequently occurring TEAEs included nausea, headache and pyrexia (Table 3). Approximately $60 \%$ of patients in this analysis experienced an opioid-related TEAE (apnea, confusion, constipation, dyspnea, hypotension, hypoventilation, hypoxia, ileus, nausea, pruritus, somnolence, tachycardia, vomiting and urinary retention); a slightly higher incidence was seen in patients with vaginal hysterectomy (fentanyl

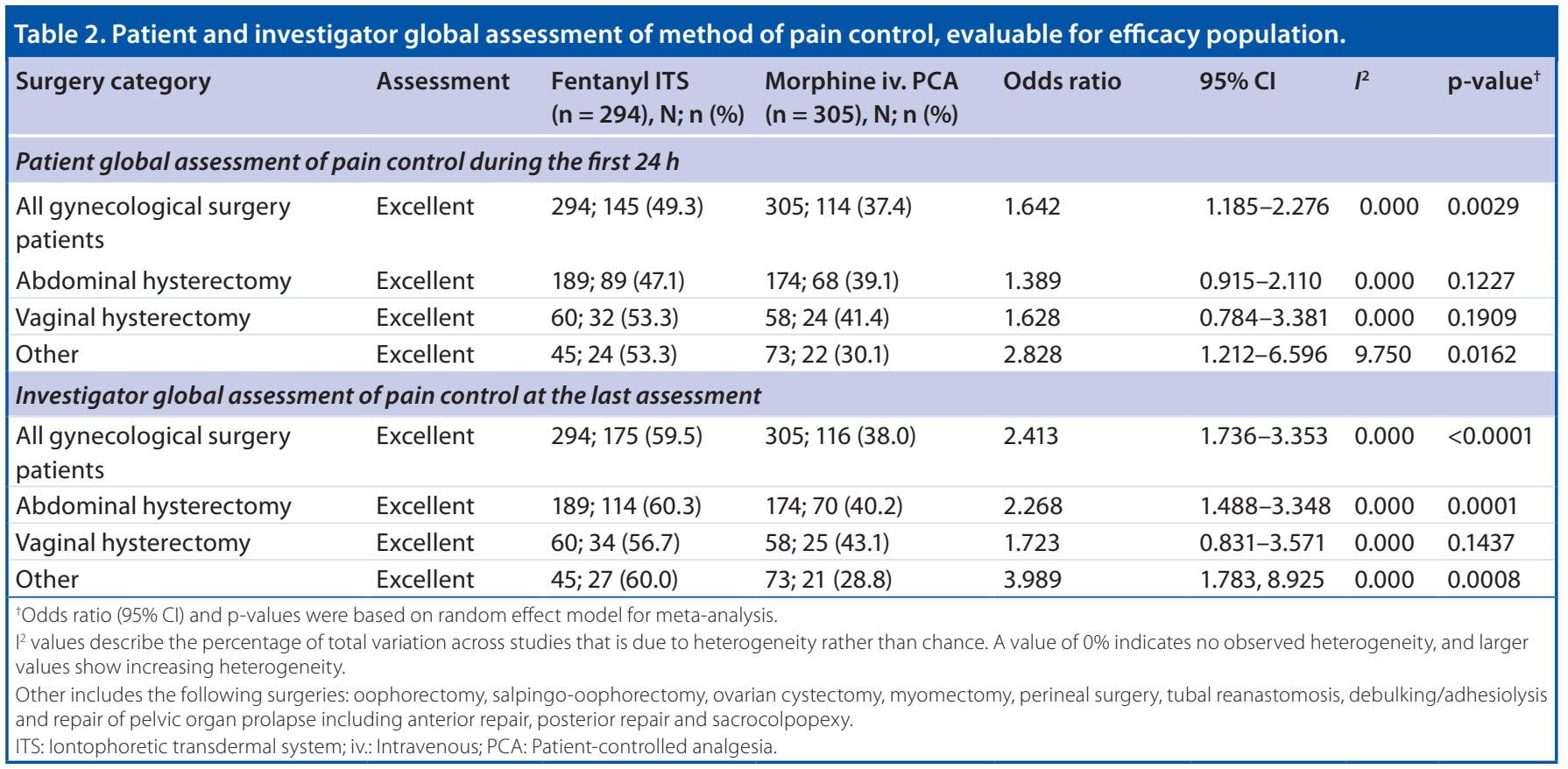


(A)

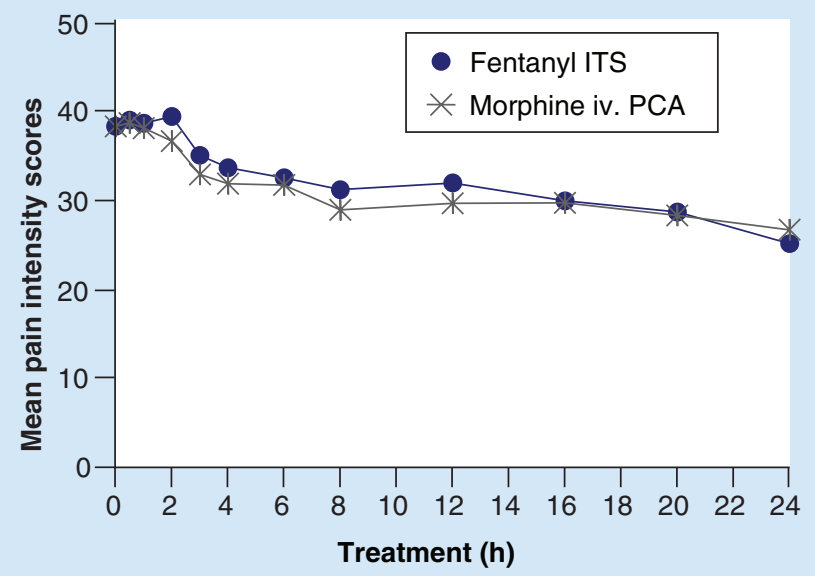

(C)

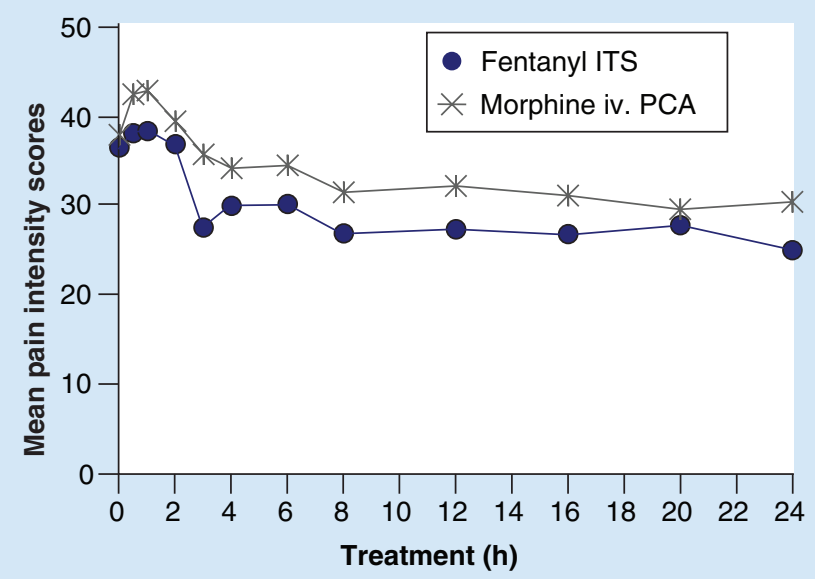

(B)

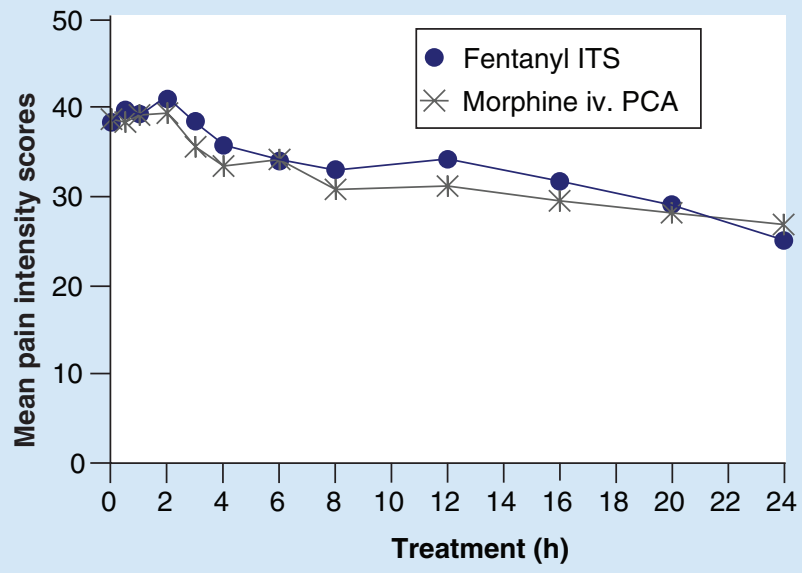

(D)

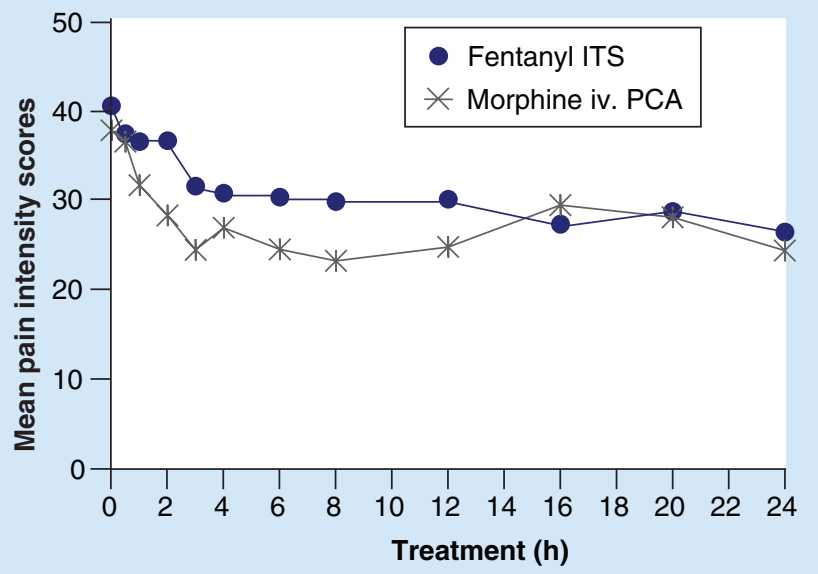

Figure 2. Pain intensity scores over time. (A) Pain intensity scores over time (all gynecological surgery patients), evaluable for efficacy population. (B) Pain intensity scores over time (abdominal hysterectomy), evaluable for efficacy population. (C) Pain intensity scores over time (vaginal hysterectomy), evaluable for efficacy population. (D) Pain intensity scores over time (other), evaluable for efficacy population. Pain intensity scores were measured on a 100-mm visual analog scale, with 0 representing 'no pain' and 100 representing 'worst possible pain' or on a numerical rating scale of $0-10$, where 0 means no pain and 10 means the worse possible pain (all numerical rating scale scores were multiplied by a factor of 10). Other includes the following surgeries: oophorectomy, salpingo-oophorectomy, ovarian cystectomy, repair of pelvic organ prolapse including anterior repair, posterior repair and sacrocolpopexy, myomectomy, perineal surgery, tubal reanastomosis and debulking/adhesiolysis.

ITS: Iontophoretic transdermal system; iv.: Intravenous; PCA: Patient-controlled analgesia.

ITS: $67.2 \%$ and morphine iv. PCA: $70.7 \%$; Table 4). The only opioid-related TEAE that was statistically different between the two groups was pruritus, which occurred more frequently in the morphine iv. PCA group (15.9\%) than in the fentanyl ITS group (9.2\%; OR: 0.537; 95\% CI: 0.324-0.888; $\mathrm{p}=0.015$ ) (Tables 3 \& 4).

\section{Discussion}

In this meta-analysis of patients who had undergone gynecological surgery, there were statistically significantly more patients in the fentanyl ITS treatment group who rated their method of pain control on the PGA as 'excellent' compared with the morphine iv. PCA group. Rothman et al. have previously validated the PGA for evaluating the method of pain control [22]. In their evaluation, they found a differentiation between PGA ratings of 'good' and 'excellent'. A rating of 'excellent' on the PGA for evaluating the method of pain control was associated with better outcomes compared 
Table 3. Summary of treatment-emergent adverse events ( $\geq 2 \%$ of patients), safety population.

\begin{tabular}{|c|c|c|c|}
\hline $\begin{array}{l}\text { Adverse event (MedDRA preferred } \\
\text { term) }\end{array}$ & $\begin{array}{l}\text { Fentanyl ITS } \\
(\mathrm{n}=295), \mathrm{n}(\%)\end{array}$ & $\begin{array}{l}\text { Morphine iv. PCA } \\
(n=309), n(\%)\end{array}$ & $\begin{array}{l}\text { p-value (Fisher's exact } \\
\text { test) }\end{array}$ \\
\hline \multicolumn{4}{|l|}{ TEAE } \\
\hline Application site erythema & $17(5.8)$ & 0 & $<0.0001$ \\
\hline Application site pruritus & $14(4.7)$ & 0 & $<0.0001$ \\
\hline Edema peripheral & 0 & $10(3.2)$ & 0.0019 \\
\hline Application site reaction & $8(2.7)$ & 0 & 0.0031 \\
\hline Headache & $59(20.0)$ & $35(11.3)$ & 0.0035 \\
\hline Application site vesicles & $7(2.4)$ & 0 & 0.0064 \\
\hline Dyspepsia & $8(2.7)$ & $3(1.0)$ & 0.1344 \\
\hline Pharyngitis & $2(0.7)$ & $7(2.3)$ & 0.1775 \\
\hline Anemia & $6(2.0)$ & $12(3.9)$ & 0.2331 \\
\hline Hypertension & $7(2.4)$ & $4(1.3)$ & 0.3736 \\
\hline Insomnia & $7(2.4)$ & $4(1.3)$ & 0.3736 \\
\hline Flatulence & $10(3.4)$ & $7(2.3)$ & 0.4660 \\
\hline Back pain & $11(3.7)$ & $8(2.6)$ & 0.4887 \\
\hline Pyrexia & $47(15.9)$ & $44(14.2)$ & 0.5715 \\
\hline Dizziness & $16(5.4)$ & $14(4.5)$ & 0.7089 \\
\hline Abdominal pain & $10(3.4)$ & $11(3.6)$ & 1.000 \\
\hline \multicolumn{4}{|l|}{ Opioid-related TEAE } \\
\hline Constipation & $9(3.1)$ & $3(1.0)$ & 0.0828 \\
\hline Hypotension & $6(2.0)$ & $5(1.6)$ & 0.7678 \\
\hline Hypoxia & $9(3.1)$ & $11(3.6)$ & 0.8218 \\
\hline Nausea & $157(53.2)$ & $158(51.1)$ & 0.6255 \\
\hline Pruritus & $27(9.2)$ & $49(15.9)$ & 0.0141 \\
\hline Vomiting & 39 (13.2) & $28(9.1)$ & 0.1200 \\
\hline Urinary retention & $6(2.0)$ & $4(1.3)$ & 0.5357 \\
\hline \multicolumn{4}{|c|}{$\begin{array}{l}\text { Any adverse events related to the application site should only occur in fentanyl ITS group hence } p<0.05 \text { is expected. } \\
\text { 'Opioid-related TEAEs include apnea, constipation, confusion, dyspnea, hypotension, hypoventilation, hypoxia, ileus, nausea, } \\
\text { pruritus, somnolence, tachycardia, vomiting and urinary retention. Only those that occurred in } \geq 2 \% \text { of patients are included in this } \\
\text { table. } \\
\text { ITS: Iontophoretic transdermal system; iv: Intravenous; PCA: Patient-controlled analgesia; TEAE: Treatment-emergent adverse event. }\end{array}$} \\
\hline
\end{tabular}

with a rating of 'good' (e.g., lower mean pain intensity scores, greater patient satisfaction, improved overall ease-of-use). Therefore, ratings of 'excellent' on the PGA may be a more relevant measure of success in postoperative pain management regimen and in this metaanalysis, fentanyl ITS is superior to morphine iv. PCA on this measurement. Investigators also rated the method of pain control as 'excellent' statistically significantly more frequently than with fentanyl ITS compared with morphine iv. PCA. Understanding that the definition of successful pain management may extend beyond the typical pain-rating scales (e.g., VAS or NRS) is critical in the current hospital environment. The Hospital Consumer Assessment of healthcare providers and systems incorporates queries regarding the level of pain and efforts made to control pain, resulting in rewards for 'top box' or excellent results. Hospitals are now financially incentivized to ensure that not only is a patient's pain well controlled but also that a patient understands that best efforts are being made to do so. This expanded focus on pain management and its incorporation into overall patient satisfaction may reward the use of new technologies like the fentanyl ITS, which have high ratings in patient-reported outcomes extending beyond level of pain.

Patient-controlled analgesia delivered via the fentanyl ITS has several benefits over the traditional iv. or epidural routes of administration. The fentanyl ITS requires no programming, which is a benefit over traditional iv. or epidural PCS. Programming the equipment associated with iv. or epidural administration is fairly complex and has been shown to be a significant source of medication errors [23-26]. The fentanyl ITS does not require an iv. that can hinder the patient's mobility. Patient mobility is an important factor 


\begin{tabular}{|c|c|c|c|c|}
\hline Surgery type & Assessment & $\begin{array}{l}\text { Fentanyl ITS } \\
(\mathrm{n}=295), \mathrm{N} ; \mathrm{n}(\%)\end{array}$ & $\begin{array}{l}\text { Morphine iv. } \\
\text { PCA ( } n=309) \\
\text { N; } n(\%)\end{array}$ & p-value ${ }^{\dagger}$ \\
\hline \multirow{3}{*}{$\begin{array}{l}\text { All gynecological surgery } \\
\text { patients }\end{array}$} & TEAE & $295 ; 240(81.4)$ & $309 ; 235(76.1)$ & 0.1136 \\
\hline & $\begin{array}{l}\text { Opioid-related TEAE. } \\
\text { Statistically different } \\
\text { opioid-related TEAE }\end{array}$ & $295 ; 183(62.0)$ & $309 ; 193(62.5)$ & 0.9332 \\
\hline & Pruritus & $295 ; 27(9.2)$ & $309 ; 49$ (15.9) & 0.0141 \\
\hline \multirow[t]{3}{*}{ Abdominal hysterectomy } & TEAE & $189 ; 153(81.0)$ & 176; $132(75.0)$ & 0.2054 \\
\hline & $\begin{array}{l}\text { Opioid-related TEAE. } \\
\text { Statistically different } \\
\text { opioid-related TEAE }\end{array}$ & $189 ; 114(60.3)$ & $176 ; 108(61.4)$ & 0.9146 \\
\hline & Pruritus & $15(7.9)$ & $31(17.6)$ & 0.0069 \\
\hline \multirow[t]{2}{*}{ Vaginal hysterectomy } & TEAE & $61 ; 48(78.7)$ & $58 ; 48(82.8)$ & 0.6461 \\
\hline & $\begin{array}{l}\text { Opioid-related TEAE. } \\
\text { Statistically different } \\
\text { opioid-related TEAE } \\
\text { None }\end{array}$ & $61 ; 41(67.2)$ & $58 ; 41(70.7)$ & 0.6973 \\
\hline \multirow[t]{3}{*}{ Other } & TEAE & $45 ; 39(86.7)$ & $75 ; 55$ (73.3) & 0.1104 \\
\hline & $\begin{array}{l}\text { Opioid-related TEAE. } \\
\text { Statistically different } \\
\text { opioid-related TEAE }\end{array}$ & $45 ; 28(62.2)$ & $75 ; 44(58.7)$ & 0.8475 \\
\hline & None & & & \\
\hline \multicolumn{5}{|c|}{$\begin{array}{l}\text { Opioid-related TEAEs included reports of apnea, constipation, confusion, dyspnea, hypotension, hypoventilation, hypoxia, ileus, } \\
\text { nausea, pruritus, somnolence, tachycardia, vomiting and urinary retention. } \\
\text { Other includes the following surgeries: oophorectomy, salpingo-oophorectomy, ovarian cystectomy, myomectomy, perineal } \\
\text { surgery, tubal reanastomosis, debulking/adhesiolysis and repair of pelvic organ prolapse including anterior repair, posterior repair } \\
\text { and sacrocolpopexy. } \\
\text { Any adverse events related to the application site should only occur in fentanyl ITS group hence p }<0.05 \text { is expected. } \\
\text { tp-value based upon Fisher's exact test comparing fentanyl ITS and morphine iv. PCA group. } \\
\text { ITS: Iontophoretic transdermal system; iv: Intravenous; PCA: Patient-controlled analgesia; TEAE: Treatment-emergent adverse event. }\end{array}$} \\
\hline
\end{tabular}

in recovery and is also very important in preventing complications following surgery (e.g., deep vein thrombosis, pneumonia and so on). In addition, without iv. lines there would not be issues with iv. line occlusion or catheter infiltrations.

Nursing ease-of-care was measured in two active-comparator trials of fentanyl ITS versus morphine iv. PCA. In both studies, nurses utilized the validated nurse ease-of-use questionnaire [27]. Significantly more nurses preferred the ITS over the iv. system in terms of overall ease-of-care, time-efficiency, convenience and satisfaction.

An internet survey of nurse and patient satisfaction that compared ITS administration versus iv. administration of PCA found that nurses preferred the ITS in terms of ease of use, safety and tolerability; however, they preferred the traditional iv. PCA for the ability to vary drugs and doses and deliver basal doses [2]. In this same survey, patients reported significantly higher satisfaction with the ITS for pain relief, safety, self-control and patient and nurse ease of care.
Overall, the TEAEs were similar between the two treatment groups. The most frequently reported TEAEs were nausea, headache, pyrexia, vomiting and pruritus. There was a statistically significant advantage in terms of pruritus in favor of fentanyl ITS and an advantage in terms of headache in favor of morphine iv. PCA. Not surprisingly, there were more application site TEAEs (i.e., erythema, pruritus and reaction) in the fentanyl ITS group.

A similar number of patients dropped out from both treatment groups; however, there were more patients who dropped out due to inadequate analgesia in the fentanyl ITS group than in the morphine iv. PCA group. It is not entirely clear why this occurred as the efficacy results were similar between the two groups and in fact, more patients in the fentanyl ITS group gave a rating of 'excellent' than in the morphine iv. PCA group. One possible explanation is the open-label design of the two clinical trials. Investigators and nurses knew the treatment being administered and may have been biased toward the treatment that they 
were familiar with (i.e., morphine iv. PCA) rather than the experimental treatment (i.e., fentanyl ITS). Therefore, they may have been less likely to discontinue morphine iv. PCA as they had a greater comfort level with this product over the unknown (at that time) fentanyl ITS.

One of the limitations of this meta-analysis is that neither study that made up the analysis was specifically designed to evaluate only patients with gynecological surgery. However, the sample size was large enough (i.e., $\mathrm{n}=604$ ) to give some confidence to the findings from these two similarly designed studies. A second limitation is that the study was not blinded. Given that the devices are so different, it would have been difficult to blind the study. In addition, a blinded study would not have yielded as much information in terms of nursing ease of care.

\section{Conclusion}

The results from this meta-analyses demonstrate that fentanyl ITS provides postoperative pain relief with good safety and tolerability in patients who have undergone gynecological surgery.

\section{Financial \& competing interests disclosure}

HS Minkowitz reports the following relevant disclosures: This research has been funded by The Medicines Company, AcelRX Pharma and Mallinckrodt. L Ding, JB Jones and $H$ Danesi are employees of The Medicines Company. The authors have no other relevant affiliations or financial involvement with any organization or entity with a financial interest in or financial conflict with the subject matter or materials discussed in the manuscript apart from those disclosed.

Writing assistance was utilized in the production of this manuscript by S Grundy.

\section{References}

Papers of special note have been highlighted as:

- of interest; $\bullet$ of considerable interest

1 Apfelbaum JL, Chen C, Mehta SS, Gan TJ. Postoperative pain experience: results from a national survey suggest postoperative pain continues to be undermanaged. Anesth. Analg. 97(2), 534-540 (2003).

2 Gan TJ, Habib AS, Miller TE, White W, Apfelbaum JL. Incidence, patient satisfaction, and perceptions of post-surgical pain: results from a us national survey. Curr. Med. Res. Opin. 30(1), 149-160 (2014).

3 Wells N, Pasero C, Mccaffery M. Improving the quality of care through pain assessment and management. In: Patient Safety and Quality: An Evidence-Based Handbook for Nurses. Hughes R (Ed.). Agency for Healthcare Research and Quality, Rockville, MD, USA (2008).

4 Weiss AJ, Elixhauser A. Trends in operating room procedures in U.S. Hospitals, 2001-2011 (statistical brief \#171). www.hcup-us.ahrq.gov

5 Smythe M. Patient-controlled analgesia: a review. Pharmacotherapy 12(2), 132-143 (1992).

6 Hudcova J, Mcnicol E, Quah C, Lau J, Carr DB. Patient controlled opioid analgesia versus conventional opioid analgesia for postoperative pain. Cochrane Database Syst. Rev. (4), CD003348 (2006).

7 Stanley TH. The history and development of the fentanyl series. J. Pain Symptom Manage. 7(3 Suppl.), S3-S7 (1992).
8 Vardanyan RS, Hruby VJ. Fentanyl-related compounds and derivatives: current status and future prospects for pharmaceutical applications. Future Med. Chem. 6(4), 385-412 (2014).

9 Rosow CE, Moss J, Philbin DM, Savarese JJ. Histamine release during morphine and fentanyl anesthesia. Anesthesiology 56(2), 93-96 (1982).

10 Grape S, Schug SA, Lauer S, Schug BS. Formulations of fentanyl for the management of pain. Drugs 70(1), 57-72 (2010).

11 Mayes S, Ferrone M. Fentanyl $\mathrm{HCl}$ patient-controlled iontophoretic transdermal system for the management of acute postoperative pain. Ann. Pharmacother. 40(12), 2178-2186 (2006).

12 Mather LE. Clinical pharmacokinetics of fentanyl and its newer derivatives. Clin. Pharmacokinet. 8(5), 422-446 (1983).

13 Chelly JE, Grass J, Houseman TW, Minkowitz H, Pue A. The safety and efficacy of a fentanyl patient-controlled transdermal system for acute postoperative analgesia: a multicenter, placebo-controlled trial. Anesth. Analg. 98(2), 427-433 (2004).

- A Phase III double-blind, placebo-controlled study of fentanyl iontophoretic transdermal system (ITS) versus placebo.

14 Viscusi ER, Reynolds L, Chung F, Atkinson LE, Khanna S. Patient-controlled transdermal fentanyl hydrochloride vs intravenous morphine pump for postoperative pain: a randomized controlled trial. JAMA 291(11), 1333-1341 (2004).
-• One of the two Phase IIIB, active-comparator trials that were utilized for this meta-analysis.

15 Hartrick CT, Bourne MH, Gargiulo K, Damaraju CV, Vallow S, Hewitt DJ. Fentanyl iontophoretic transdermal system for acute-pain management after orthopedic surgery: a comparative study with morphine intravenous patient-controlled analgesia. Reg. Anesth. Pain Med. 31(6), 546-554 (2006).

- A Phase IIIB randomized, active-comparator trial comparing fentanyl ITS with morphine intravenous patient-controlled analgesia.

16 Viscusi ER, Reynolds L, Tait S, Melson T, Atkinson LE. An iontophoretic fentanyl patient-activated analgesic delivery system for postoperative pain: a double-blind, placebo-controlled trial. Anesth. Analg. 102(1), 188-194 (2006).

- A Phase III double-blind, placebo-controlled study of fentanyl ITS versus placebo.

17 Minkowitz HS, Rathmell JP, Vallow S, Gargiulo K, Damaraju CV, Hewitt DJ. Efficacy and safety of the fentanyl iontophoretic transdermal system (ITS) and intravenous patient-controlled analgesia (IV PCA) with morphine for pain management following abdominal or pelvic surgery. Pain Med. 8(8), 657-668 (2007).

-. One of the two Phase IIIB, activecomparator trials that were utilized for this meta-analysis.

18 Grond S, Hall J, Spacek A, Hoppenbrouwers M, Richarz U, Bonnet F. Iontophoretic transdermal system using 
fentanyl compared with patient-controlled intravenous analgesia using morphine for postoperative pain management. $\mathrm{Br}$. $\mathrm{J}$. Anaesth.98(6), 806-815 (2007).

- A Phase IIIB randomized, active-comparator trial comparing fentanyl ITS with morphine intravenous patient-controlled analgesia.

19 Markey DW, Brown RJ. An interdisciplinary approach to addressing patient activity and mobility in the medical-surgical patient. J. Nurs. Care Qual. 16(4), 1-12 (2002).

20 Morris BA, Benetti M, Marro H, Rosenthal CK. Clinical practice guidelines for early mobilization hours after surgery. Orthop. Nurs.29(5), 290-316 (2010)

21 Lindley P, Pestano CR, Gargiulo K. Comparison of postoperative pain management using two patient-controlled analgesia methods: nursing perspective. J. Adv. Nurs. 65(7), 1370-1380 (2009).

22 Rothman M, Vallow S, Damaraju CV, Hewitt DJ. Using the patient global assessment of the method of pain control to assess new analgesic modalities in clinical trials. Curr. Med. Res. Opin.25(6), 1433-1443 (2009).

- This article represents the validation of the patient global method of pain control.

23 Hicks RW, Sikirica V, Nelson W, Schein JR, Cousins DD. Medication errors involving patient-controlled analgesia. Am. J. Health Syst. Pharm.65(5), 429-440 (2008).

24 Schein JR, Hicks RW, Nelson WW, Sikirica V, Doyle DJ. Patient-controlled analgesia-related medication errors in the postoperative period: causes and prevention. Drug Saf. 32(7), 549-559 (2009).

25 Institute for Safe Medication Practices. High alert medication feature: reducing patient harm from opiates.

www.ismp.org

26 Institute for Safe Medication Practices. Misprogramming PCA concentrations leads to dosing errors. www.ismp.org

27 Harding G, Vallow S, Leidy NK et al. Ease of care with patient controlled analgesia systems: questionnaire development and validation. J. Adv. Nurs. 59(5), 530-541 (2007). 